\title{
A Model to Guide Telehealth Research Quality Improvement, Practice and Education
}

\author{
Jane M CARRINGTON, PhD, RN, FAAN, FAMIA a and \\ Rene LOVE, PhD, DNP, PMHNP-BC, FNAP, FAANP ${ }^{\mathrm{a}}$ \\ ${ }^{a}$ University of Florida, College of Nursing, Gainesville, Florida, USA
}

\begin{abstract}
Telehealth is a means for providing care to our rural patients. This mode of healthcare delivery is not without challenges with technology, research, quality improvement, practice and education. Here we present the TRIP-E Meta Model. This model was derived from a two phased process. First, we performed a review of literature and from this developed the first attempt at model development. Second, we took student evaluations from a telehealth education program for Doctor of Nursing Practice students. Based on their feedback, we then further advanced the first model to the TRIP-E Meta Model. This model is a comprehensive guide to research, quality improvement, practice and education. As a meta model, the TRIPE can have other theories applied for projects. This feature of meta models provide flexibility for the model. We invite others to test this model for its flexibility and usability.
\end{abstract}

Keywords. Telehealth, model, informatics, quality improvement

\section{Introduction}

Within the United States, 60 million people or $25 \%$ of the population live in what is defined as rural America according to the US Census Bureau. Rural areas contain fewer resources for those who living with mental and physical chronic conditions. Telehealth is a means for addressing this gap with connected care. As telehealth solutions become more available to rural residents, scientists, informatics specialists, providers, and educators are challenged to adopt a suitable theory. We developed the TRIP-E Meta Model to meet this gap. This model is innovative as it addresses each of these perspectives of telehealth and allows for application of other theories within the meta model. Here we present the process used to develop the model and introduce the TRIPE Meta Model.

\section{Methods}

The TRIPE Meta Model is the result of a two phased project process. First, was a review of literature and second, was feedback from Doctor of Nursing Practice students from a telehealth module. Both will be further discussed herein. 


\subsection{Review of Literature}

Authors performed a search of the literature using keywords "Telehealth" and "Models" and "Framework" and common search engines: Google Scholar, CINHL, and PubMed. No limitations were placed on publication years. Inclusion criteria included printed in English, research papers, and published in peer reviewed journals. Results from this search were 1,080 papers from which six papers emerged as being directly related to the goals of the project. These models or frameworks were then assessed for research, practice, quality improvement, and education.

Each paper described a process of model development from concept development, focus groups, known informatics processes, policy foundation, and comparisons of frameworks $[1,2,3,4,5,6]$. Not surprising researchers would approach framework development for telehealth using principles of informatics from user experience [1] to staged implementation [3]. Researchers also sought insights from patients or end users [2] and policy experts for model development [4]. Two papers described framework development using a synthesis of literature [5 and 6].

The frameworks that evolved from the above processes focused on aspects of telehealth, rather than the complete process for connected health. Each framework is suitable for a portion of the process for implementation $[1,3,6]$, communication [2,3], or policy development $[4,5]$. We determined these frameworks each lacked components that would be essential for an encompassing a framework for comprehensive connected health.

\subsection{Program Evaluation}

We developed a telehealth educational module for Doctor of Nursing Practice (DNP) students at the University of Arizona. Eighty-three students from Adult Gero Acute Care, Psych Mental Health, Pediatric, and Family Nurse Practitioner programs took part in the first offering of the module. Of these, 78 (94\%) completed the evaluation survey. Consistently, students commented that they appreciated the program, however, they struggled with the technology, patient visualization, and not performing "hands on" assessment.

Based on the student evaluations, we determined that the constructs training and evaluation should be included and with demonstrated relationships with providers and patients. The program evaluation did not suggest the alteration of previously included constructs or their relationships.

\section{Results}

Here we presented the results from both the literature review and program evaluation. From the review of literature, we learned that the emphasis of telehealth frameworks is implementation, communication, and policy development. From this we developed the Telehealth Framework (Figure 1) [10]. This framework includes patient data from their history and monitor data that includes home monitoring and sensors. The framework includes technology and the interface between provider and patient end users. The intervention comes from technology and care team and patient partnership towards an outcome. The outcomes are evaluated back to the patient data. 


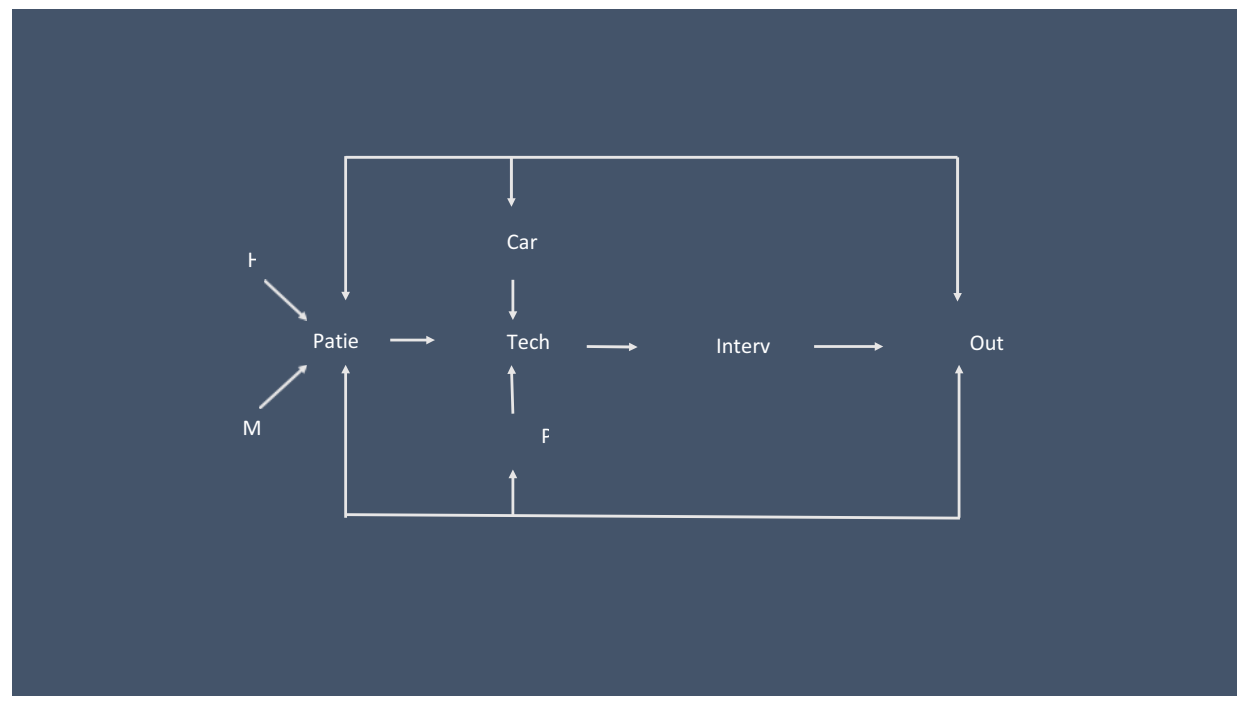

Figure 1. Telehealth framework [7].

Program evaluation data from students revealed missing constructs Training and Evaluation (Figure 2). We included the constructs Training and Evaluation with the relationship to the provider and patient signifying importance of education to the success of telehealth programs. Equally important is the evaluation of the training for both the providers and patients. We also changed the constructs History and Monitor to Health Record and Monitoring and Sensors. Both constructs are now broader to account for the movement toward big data and data science in telehealth. Also, these changes allow for a model that can guide telehealth through the lifespan and allows broader context for care from acute to primary and rehabilitation.

The revised framework is the Telehealth Research Quality Improvement Practice Education or TRIP-E Meta Model and includes the elements of the previously described model as well as terms associated with education and is now suitable to guide telehealth research, quality improvement, practice and education. As a meta model, this model can have frameworks applied to explore the constructs and relationships.

The TRIP-E Meta Model can be used to generate knowledge via research and implement findings into practice via quality improvement. This model can also be used to guide practice, reminding the providers that the patient is a member of the care team and their data is essential in care planning as is implementation and outcomes measures. Training and evaluation added to the model implies that this is an ongoing process, not only during implementation. This framework can then also be used to guide curriculum development for teaching students to become telehealth providers. As a meta model, those who adopt this framework can apply theories to specifically address variables in each phase of the model. 


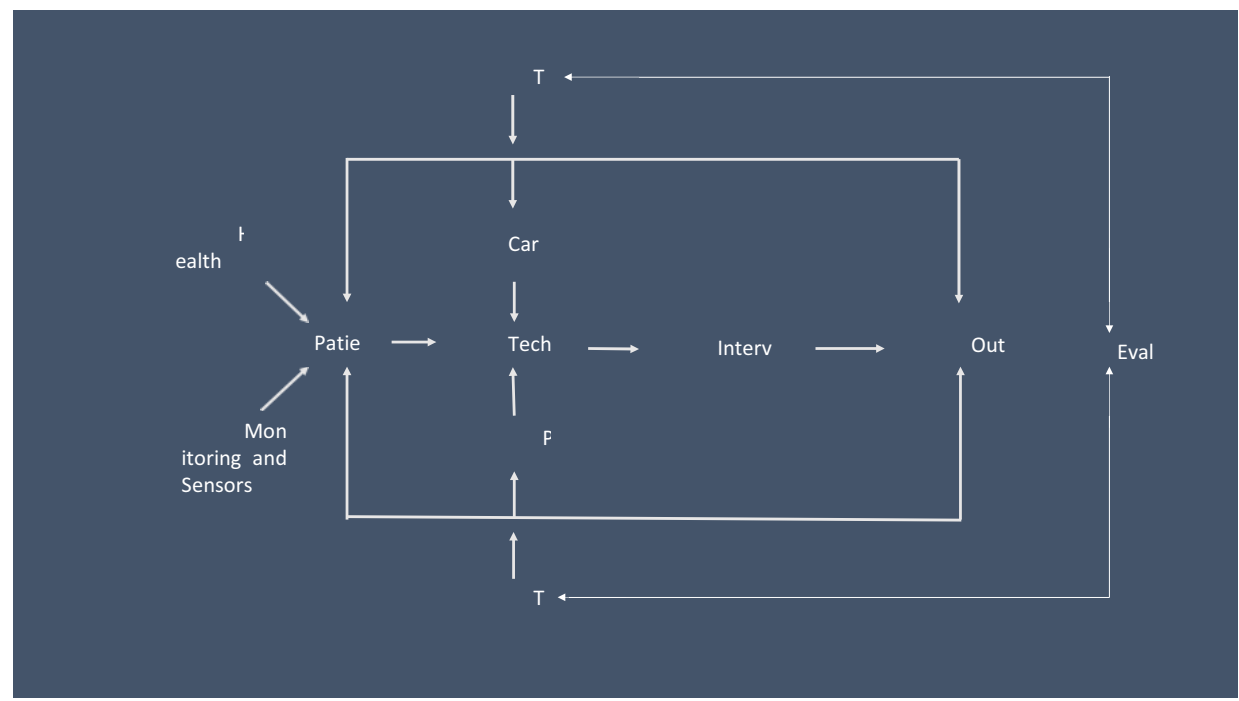

Figure 2. TRIP-E meta model.

\section{Summary}

Here we have presented the TRIP-E Meta Model's derivation. We began with a review of literature and then program evaluation of students' participation in a telehealth program of learning. From this feedback the TRIP-E Meta Model emerged and is ready for testing. With testing, we are confident that the TRIP-E Meta Model will support the development of additional middle range frameworks useful to continue exploration of telehealth and patient outcomes.

Implications for connected care with TRIP-E is the existence of a comprehensive model to guide telehealth. The goal in using telehealth strategies is to connect patients to primary and specialty care services.

Future work includes the adoption of TRIP-E Meta Model for preparation of researchers to explore and generate knowledge to increase patient outcomes, informatics specialists to perform and guide quality improvement projects to improve practice and technology, and students to serve as providers using telehealth.

\section{References}

[1] Hensel BK, Demiris G, Courtney KL. Defining obtrusiveness in home telehealth technologies: A conceptual framework. Journal of the American Medical Informatics Association, 13 (2006), 428-431.

[2] Hopp FP, Hogan M. Community-based tele-health systems for persons with diabetes: Development of an outcomes model. Social Work in Health Care, 48 (2009), 134-153.

[3] Othermani M, Diouny S, Boumalif O. The application of a telemedicine management system: The case of a Moroccan hospital unit. Assistive Technolgy. 24 (2012).209-213.

[4] Edmunds M, Tuckson R, Lewis J et al. An emergent research and policy framework for telehealth. eGEMs. 5 (2017). 1-10.

[5] Van Dyk L. A review of Telehealth Service Implementation Frameworks. International Journal of Environmental Research and Public Health. 11 (2014). 1279-1298. 
[6] Nepal S, Jang-Jaccard, Alem L. Case study: A framework for telehealth program evaluation. Telemedicine and e-Health 20 (2014), 393-404.

[7] Love R, Johnson D, Carrington JM. Critical conversations about telehealth in the 21 st century for psychiatric mental health nurse practitioners. International Society of Psychiatric-Mental Health Nurses, Charlotte, NC. 2019. 